\title{
Autophagy and bacterial infectious diseases
}

\author{
Jae-Min Yuk ${ }^{1,2}$, Tamotsu Yoshimori ${ }^{3}$ \\ and Eun-Kyeong $\mathrm{Jo}^{1,2,4}$ \\ ${ }^{1}$ Department of Microbiology and \\ ${ }^{2}$ Infection Signaling Network Research Center \\ Chungnam National University School of Medicine \\ Daejeon 301-747, Korea \\ ${ }^{3}$ Department of Genetics \\ Graduate School of Medicine \\ Osaka University \\ Osaka 565-0871, Japan \\ ${ }^{4}$ Corresponding author: Tel, 82-42-580-8243; \\ Fax, 82-42-580-8436; E-mail, hayoungj@cnu.ac.kr \\ http://dx.doi.org/10.3858/emm.2012.44.2.032 \\ Accepted 30 December 2011 \\ Available Online 19 January 2012
}

Abbreviations: ATG, autophagy-related protein; eis, enhanced intracellular survival; GABARAP, $\gamma$-aminobutyric acid receptorassociated protein; GAS, group A Streptococcus; Gbp, guanylatebinding protein; LC3, light chain 3; M. tuberculosis, Mycobacterium tuberculosis; NF, nuclear factor; PAMP, pathogen associated molecular pattern; ROS, reactive oxygen species reactive oxygen species; SCVs, Salmonella-containing vacuoles; SLAPs, spacious Listeria-containing phagosomes; $S$. Typhimurium, Salmonella enterica serovar Typhimurium; T3SSs, type three secretion systems; Th, T helper; TRAF6, tumor necrosis factor receptor-associated factor 6

\begin{abstract}
Autophagy is a housekeeping process that maintains cellular homeostasis through recycling of nutrients and degradation of damaged or aged cytoplasmic constituents. Over the past several years, accumulating evidence has suggested that autophagy can function as an intracellular innate defense pathway in response to infection with a variety of bacteria and viruses. Autophagy plays a role as a specialized immunologic effector and regulates innate immunity to exert antimicrobial defense mechanisms. Numerous bacterial pathogens have developed the ability to invade host cells or to subvert host autophagy to establish a persistent infection. In this review, we have summarized the recent advances in our understanding of the interaction between antibacterial autophagy
\end{abstract}

(xenophagy) and different bacterial pathogens.

Keywords: autophagy; cytokines; immunity, Innate; infection; reactive oxygen species

\section{Introduction}

During macroautophagy (herein referred to as autophagy), cytoplasmic contents can be sequestered in a unique double-membrane structure, the autophagosome. Through autophagy, cytoplasmic cargo in autophagosomes can be degraded by fusion of autophagosomes and lysosomes, and activation of the lysosomal degradation pathway (Levine and Deretic, 2007). In addition to degradation of damaged organelles or materials, autophagy has received attention as a crucial component of innate defense against a variety of infectious agents. Autophagy of foreign micro-organisms, i.e., xenophagy, has emerged as a powerful method of eliminating intracellular bacteria (Gutierrez et al., 2004; Nakagawa et al., 2004). However, there is a complex interplay between host autophagy mechanisms and pathogens. Numerous microorganisms have evolved strategies to evade or subvert host autophagy to survive and establish a persistent infection (Campoy and Colombo, 2009b; Orvedahl and Levine, 2009). Thus, identification of mechanisms or virulence factors exploiting autophagy may provide a new strategy for therapeutic intervention in infectious diseases.

Host defense against pathogens requires coordination of multiple innate immune signaling pathways (Jo et al., 2007). During infection, host cells recognize the pathogen-associated molecular patterns of a variety of microbes through the expression of various pattern recognition receptors. The recognition of foreign or danger molecules by innate receptors can trigger an intracellular signaling cascade, leading to activation of antimicrobial effector mechanisms to promote clearance of the infection (Jo et al., 2007; Brodsky and Medzhitov, 2009). Autophagy is considered to be one of the effector mechanisms downstream of these receptors, and plays an integral role in both innate and adaptive immunity to various pathogens (Orvedahl and Levine, 2009).

Autophagy also crosstalks with intracellular sig- 
naling molecules and effectors (Brodsky and Medzhitov, 2009; Shahnazari and Brumell, 2011). Through these interactions, autophagy carries out not only direct microbial degradation but also other protective mechanisms, such as lysozyme secretion, the ubiquitin-mediated pathway, and antigen presentation (Brodsky and Medzhitov, 2009; Shahnazari and Brumell, 2011). In particular, the autophagic or cargo receptors have received much attention, since they play an important role in transporting ubiquitinated microbial cargos to the autophagy machinery (Pankiv et al., 2007; Ichimura et al., 2008; Kirkin et al., 2009b; Thurston et al., 2009; von Muhlinen et al., 2010).

Recent studies have provided evidence that autophagy acts as a 'tuning module' in the regulation of innate immunity to prevent excessive inflammatory responses and inflammasome signaling (Sumpter and Levine, 2010). Generation of reactive oxygen species (ROS) of cellular and mitochondrial origin is thought to play an important role in autophagy regulation, thus influencing innate defense (Azad et al., 2009; Scherz-Shouval and Elazar, 2011).

The specific role and regulatory mechanisms of autophagy in connection with innate immune pathways during infection will be discussed here. Additionally, the molecular mechanisms by which multiple bacteria and viruses evade and/or resist autophagy will be covered in this review.

\section{Xenophagy: autophagic control of intracellular bacterial pathogens}

Autophagy is a mechanism for adjusting cellular quality and quantity through capture and degradation of portions of the cytosol or organelles in response to diverse stress or stimuli, including infection (Deretic and Levine, 2009). Autophagy is a means of bulk degradation of cytoplasmic components within lysosomes. During this process, cytoplasmic constituents are delivered to lysosomes for degradation (Mizushima and Levine, 2008). Although autophagy is fundamentally a 'self-eating' process, it also facilitates degradation of pathogens as a 'xenophagy' process (Huang and Brumell, 2009).

Accumulating evidence indicates that autophagy acts as a defense mechanism against multiple invading microbes (Deretic and Levine, 2009). During infection, host autophagic responses can target several steps of bacterial invasion. For example, autophagy can target bacteria within vacuolar membranous compartments. Intracellular pathogens, such as Mycobacterium tuberculosis (M. tuberculosis), can actively survive within host cells and exploit host defense by inhibition of phagosomal maturation (Gutierrez et al., 2004; Yuk et al., 2009b). Autophagy activation by nutrient starvation or rapamycin treatment leads to co-localization of mycobacterial phagosomes with autophagosomes to overcome the inhibition of phagosomal maturation by mycobacteria, thereby resulting in suppression of intracellular bacterial survival (Gutierrez et al., 2004; Yuk et al., 2009b). Stimulation of host autophagy by treatment with vitamin $D$ significantly enhanced the antimicrobial responses against $M$. tuberculosis in human macrophages. This effect was mediated by cathelicidin, a peptide that is induced by vitamin $\mathrm{D}$ and promotes co-localization of bacterial phagosomes and autophagosomes (Yuk et al., 2009b). These data suggest that autophagy contributes to elimination of bacteria by overcoming bacterial resistance to lysosomal access.

Another example is group A Streptococcus (GAS). When GAS enters epithelial cells via endocytosis, they can escape from the endosomes to the cytoplasm by secretion of streptolysin $\mathrm{O}$, a poreforming toxin (Nakagawa et al., 2004). GAS is then trapped in autophagosome-like compartments, which mature into autolysosomes and so eliminate the bacteria (Nakagawa et al., 2004). Mechanistically, the host small G proteins, Rab5 and Rab7, are associated with autophagosome formation and the progression of endosome maturation in cells infected with GAS (Sakurai et al., 2010). Two endocytic soluble $\mathrm{N}$-ethylmaleimide-sensitive factor attachment protein receptors, VAMP8 from lysosomes and Vti1b from autophagic compartments, are required for the successful fusion of GAS-containing xenophagosomes with lysosomes, which facilitates xenophagy (Furuta et al., 2010).

The third mode of anti-bacterial action of autophagy is receptor-mediated recruitment of bacteria to autophagosomes. Some populations of Salmonella enterica serovar Typhimurium (S. Typhimurium) are known to invade mammalian cells and replicate in Salmonella-containing vacuoles (SCVs). During this process, two kinds of Salmonella pathogenicity island (SPI) encoding type III secretion systems (T3SSs) are involved: the SPI-2 encoded T3SS facilitates bacterial growth, whereas the SPI-1 encoded T3SS causes damage to SCV (Birmingham et al., 2006), which activates the autophagic process. The autophagy system targets the intracellular bacteria present within damaged SCVs, and contributes to restriction of bacterial growth during infection (Birmingham et al., 2006). The autophagy receptor p62/SQSTM1, an adaptor protein for degradation of cargo tagged with ubiquitinated protein, is recruited to autophagy-targeted S. Typhimurium and required for the 
restriction of intracellular bacterial replication through induction of efficient antibacterial autophagy (Zheng et al., 2009). The autophagy receptor NDP52 can also target distinct compartments in cells infected with Salmonella, and is required for autophagy activation (Cemma et al., 2011). These data suggest that xenophagy is required for efficient targeting and killing of cytosolic pathogens. The post-translational modification (ubiquitination) of intracellular pathogens and the roles of cargo receptors will be discussed in detail in the latter part of this review.

\section{Cross-talk of autophagy with innate immune signaling pathways}

Apart from its initial description, which is that autophagy is a response to amino acid deprivation, to recycle cytoplasmic constituents and synthesize energy for cell survival under stressful/starved conditions, autophagy is also being recognized as an innate immune process that is activated upon recognition of microbial infection (Brodsky and Medzhitov, 2009; Shahnazari and Brumell, 2011). Bacteria and viruses possess molecules termed 'pathogen associated molecular patterns' (PAMPs) that bind to pattern-recognition receptors such as toll-like receptors (TLRs) on innate immune cells. Ligation of TLRs with PAMPs is known to activate innate immune cells and subsequently facilitate adaptive immune responses. So far, 10 TLRs in humans and 12 in mice have been identified. On the basis of subcellular localization, TLRs can be divided into extracellular and intracellular receptors: TLR1, 2, 4, 5, 6, and 10 are located on the cell surface. In contrast, TLR3, 7, 8, and 9 are expressed in intracellular endosomal/lysosomal compartments and the endoplasmic reticulum (Manavalan et al., 2011). Bacterial PAMPs are recognized by five TLRs in humans: lipopolysaccharide (LPS) is the main ligand of Gram-negative bacteria for TLR4; lipotechoic acid and diacylated lipopeptides are sensed by TLR2/6; triacylated lipopeptides are sensed by TLR2/1; CpG motifs are sensed by TLR9; and flagellin is sensed by TLR5 (Takeuchi and Akira, 2010). Interestingly, stimulation of TLR2, 4 , and 7 with their ligands can stimulate autophagy, which functions to eliminate intracellular mycobacteria (Xu et al., 2007; Shi and Kehrl, 2008; Delgado and Deretic, 2009; Shin et al., 2010b). Thus, TLR responses, which are well-known immune-activating signals, can also directly activate autophagy resulting in killing of invading bacteria.

In TLR signaling, several TIR domain-containing adaptor molecules, such as MyD88, TIRAP, and
TRIF, lie downstream of TLR and deliver activation signals. (Takeda and Akira, 2004). Upon stimulation of TLR4 with LPS, MyD88, TRIF, and tumor necrosis factor receptor (TNFR)-associated factor 6 (TRAF6), associate with ubiquitinated autophagy-related protein (Atg)6/Beclin-1, which results in activation of autophagy (Shi and Kehrl, 2010). The roles of further downstream signaling molecules of the TLR signaling pathway in the regulation of autophagy remain to be determined. Among them, the role of nuclear factor $(N F)-\kappa B$, an important downstream mediator of TLR signaling (Takeda and Akira, 2004), in autophagy is still being debated. Although the NF-KB signaling pathway inhibits the autophagy pathways in tumor cells (Djavaheri-Mergny et al., 2006) and keratinocytes (Lee et al., 2011), this has not been confirmed in primary innate cells, such as macrophages or dendritic cells. Mitogen-activated protein kinase signaling pathways may play a role in autophagy, since they are important in the activation of innate immune signaling during bacterial infection (Jo et al., 2007). The stress-activated signaling molecule, c-Jun N-terminal protein kinase 1 (JNK1), pathway has been known to activate starvation- or ceramide-induced autophagy through $\mathrm{Bcl}-2$ phosphorylation and subsequent dissociation of $\mathrm{Bcl}-2$ from Beclin-1 (Wei et al., 2008; Pattingre et al., 2009). More comprehensive data are necessary to gain a complete understanding of the roles and regulatory mechanisms of signaling modules in antibacterial autophagic activation.

Stimulation of other pattern recognition receptors, including the cytosolic receptor NOD2, regulates autophagy. NOD2 receptor recognition by muramyldipeptide induces autophagy in dendritic cells through receptor-interacting serine-threonine kinase2, ATG5, ATG7 and ATG16L1 (Cooney et al., 2010). Notably, NOD2-induced autophagy activation is required for both direct antimicrobial activities and antigen presentation to $\mathrm{T}$ cells via $\mathrm{MHC}$ class II antigen to induce adequate $\mathrm{CD} 4+\mathrm{T}$ cell responses (Cooney et al., 2010). These studies suggest that specific PAMP recognition through membrane and cytosolic innate receptors can regulate innate and adaptive immune responses through autophagy activation.

\section{Immune effectors and autophagy regulation: cytokines, IRG proteins (p47 GTPases), and cathelicidins}

Numerous cytokines and chemokines, such as interleukin (IL)-12, IL-23, IL-17, interferon (IFN)- $\alpha$, IFN- $\beta$, IFN- $\gamma$, IL-10, IL-27, IL-6, TNF, Iymphotoxin, and IL-1 family cytokines, are produced by innate 


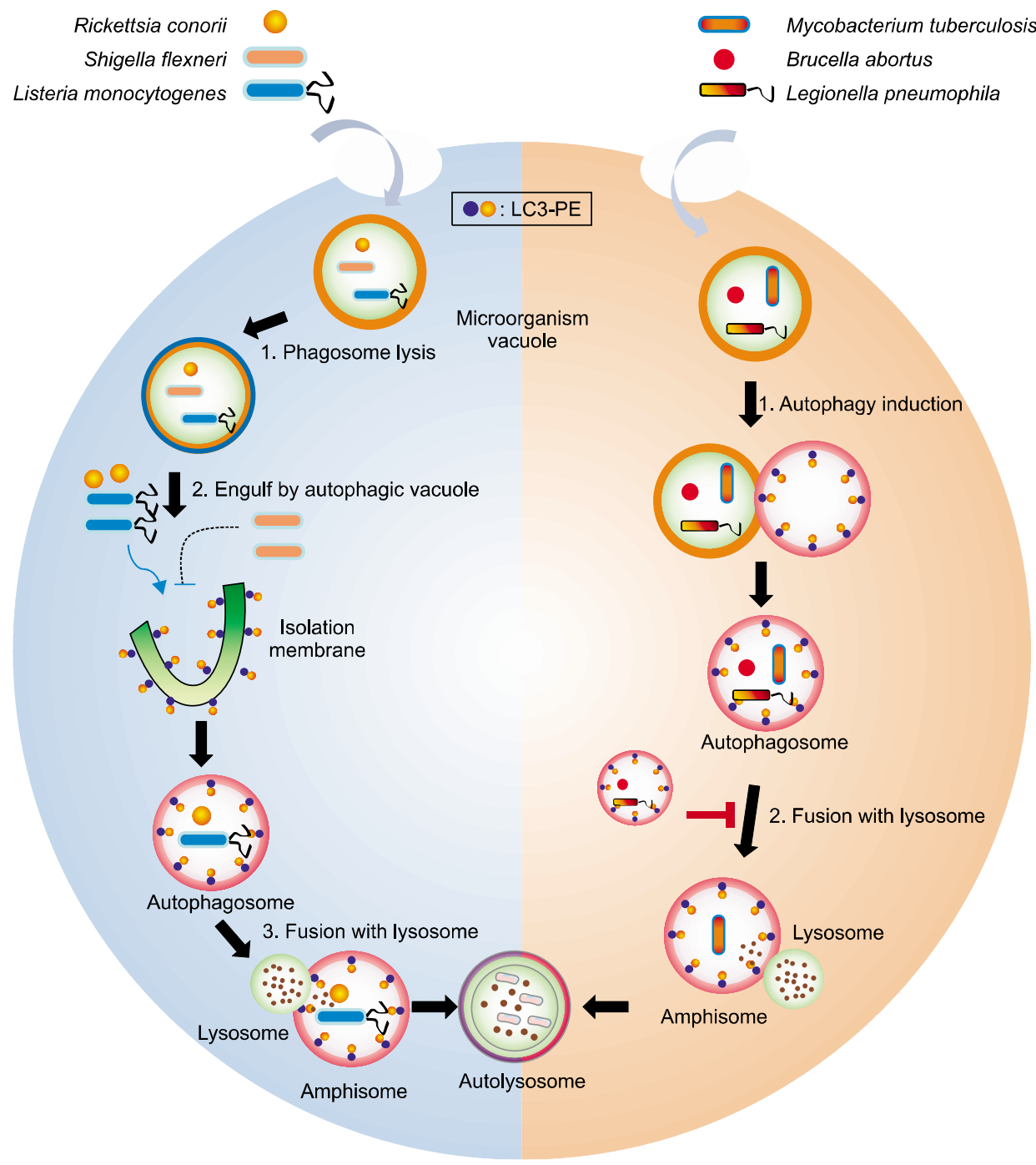

Figure 1. The role of autophagy in control of innate immunity and the survival mechanisms of intracellular bacteria. Various intracellular bacteria are internalized by phagocytes; these survive in the cytosol or phagosome through various strategies. (1) First, some bacteria are able to escape from the phagosome by secreting factors and replicating in the host cytoplasm (left panel). When autophagy is induced, Listeria monocytogenes and Rickettsia conorii present in the cytoplasm are captured by the autophagosomal membrane and degraded by the autolysosome; however, Shigella flexneri has the ability to avoid import into the autophagosome. (2) Second, some bacteria reside in the phagosomal compartment (right panel). Upon autophagy activation, phagosomes containing Mycobacterium tuberculosis are caught by the autophagic vacuole and then degraded by fusion with the lysosome. In contrast, Brucella abortus and Legionella pneumophila can inhibit autophagosome maturation through inhibition of lysosome fusion, allowing the pathogen to survive and replicate.

immune cells during bacterial infection (Cooper et al., 2011; Deretic, 2011). Notably, several cytokines are key regulators of autophagy pathways (Deretic 2009). For example, the Th1 cytokine IFN- $\gamma$ is essential for host defense against intracellular pathogens. IFN- $\gamma$ can overcome the blocking of phagosomal maturation induced by $M$. tuberculosis through autophagy activation (Gutierrez et al., 2004; Harris et al., 2009). In contrast, the T helper (Th) 2 cytokines IL-4 and IL-13 contribute to inhibition of autophagy and autophagy-mediated suppression of mycobacteria in macrophages (Harris et al., 2007).

The mechanisms by which IFN- $\gamma$ activates autophagy are: IFN- $\gamma$ activates several anti-bacterial proteins involved in autophagy, such as IRG (p47 GTPases) and the 65-kilodalton (kD) guanylatebinding protein (Gbp) gene family members (Gbp1, Gbp6, Gbp7, and Gbp10). The IRG proteins (p47
GTPases), transcription of which is induced by IFN- $\gamma$ stimulation, play an essential role in the elimination of intracellular pathogens in mice (Deretic, 2009). Murine IRG proteins are involved in innate defense against $M$. tuberculosis and Toxoplasma gondii (Gutierrez et al., 2004; Ling et al., 2006). Although the single human IRG member, IRGM, is not induced by IFN- $\gamma$, it is essential for induction of autophagy in response to intracellular bacterial infection (Singh et al., 2006). Moreover, recent studies have revealed that the Gbp gene family members (Gbp1, Gbp6, Gbp7, and Gbp10) are involved in oxidase-dependent killing and autophagy pathways in phagocytes to combat listerial or mycobacterial infection. Thus, $65-\mathrm{kD}$ Gbps function to coordinate a potent oxidative and vesicular trafficking program to protect the host from infection (Kim et al., 2011). Reciprocally, autophagy facilitates IFN- $\gamma$-activated Jak2-STAT1 
pathways and inflammatory responses, suggesting a link between autophagy and cellular inflammation (Chang et al., 2010).

Antimicrobial peptides are major components of the bactericidal machinery of mammalian phagocytes, and play an important role as innate effectors during bacterial and viral infections (Cederlund et al., 2011). Of the antimicrobial peptides, cathelicidins exert antimicrobial activities against mycobacteria (Liu et al., 2006; Yang et al., 2009; Sonawane et al., 2011). Interestingly, vitamin D activates antimycobacterial autophagy through induction of cathelicidin, which contributes to the promoter activation of the autophagy-related genes Atg5 and Beclin-1, as well as co-localization of bacterial phagosomes and autophagosomes (Yuk et al., 2009b). Future studies are necessary to fully elucidate the roles of the numerous antimicrobial peptides in the context of autophagy activation leading to collaboration with innate immunity.

\section{Bacterial tactics to modulate host autophagy}

Intracellular bacterial pathogens have developed multiple strategies for exploiting or hijacking eukaryotic functions in order to survive and multiply inside host cells (Knodler et al., 2001). Consistently, numerous pathogens antagonize the initiation and maturation of autophagic processes, evade autophagic recognition, or hijack the autophagy pathway to facilitate intracellular survival or replication (Levine et al., 2011).

The various strategies used by bacterial pathogens are summarized in Figure 1. In this section, we will summarize two strategies by which bacterial pathogens can escape from or interact with autophagic pathways: 1) physical escape from degradative endocytic compartments and replication within the cytoplasm (Shigella, Listeria, Rickettsia); and 2) survival and replication within membranebound compartments (Mycobacteria, Salmonella, Francisella, Legionella, Brucella, Chlamydia, Porphyromonas gingivalis).

\section{Bacterial escape into cytosol after breaking the host jail (bacterial endocytic compartments): Shigella, Rickettsia, M. marinum, and Listeria}

Intracellular bacteria such as Shigella flexneri and Listeria monocytogenes can evade autophagic recognition, survive, and replicate in phagocytic and non-phagocytic cells by disrupting vacuoles and escaping into the cytoplasm (Ogawa et al., 2005), and acquisition of actin-based motilities
(Cossart, 2000), respectively. S. flexneri can block autophagic targeting through the secreted T3SS effector icsB that competes with the autophagy protein Atg5 for binding to the $S$. flexneri surface protein VirG (Ogawa et al., 2005). Thus, icsB deletion mutants are not able to survive in host cells (Ogawa et al., 2005). When S. flexneri breaks the vacuolar membrane and escapes from the phagosome, the vacuolar membrane fragments are polyubiquitinated, and targeted to autophagic degradation through p62 accumulation on membrane remnants (Dupont et al., 2009).

Rickettsia are Gram-negative obligate intracellular bacteria, and can be transmitted to humans via arthropod vectors (Azad and Beard, 1998). After infection, Rickettsia can escape from phagosomes into their eukaryotic host cytoplasm, where they replicate and infect adjacent cells (Silverman and Wisseman, 1979; Walker, 2007). Earlier studies reported that rickettsial infection of polymorphonuclear leukocytes results in autophagosome formation (Rikihisa, 1984). The detailed mechanisms by which Rickettsia are able to escape from autophagy machinery remain to be determined. Conversely, the host autophagy induced during rickettsial infection is recognized as an important defense mechanism against this invading pathogen (Walker, 2007; Deretic, 2010). One report indicated that Rickettsia-induced proinflammatory cytokines led to increased activation of the autophagy that is required for bacterial inhibition (Walker et al., 1997).

Mycobacterium marinum, a natural pathogen, and the closest relative of $M$. tuberculosis with a homology of $99.4 \%$ (Stamm and Brown, 2004), has been shown to escape from the phagosome and enter the cytosol via an ESX-1-dependent mechanism (Collins et al., 2009). In the cytosol, some M. marinum promote motility through actin polymerization, while non-motile bacteria are decorated by ubiquitin and sequestered in the host Lamp1-positive vesicular compartments by autophagosome-like double membranes, formation of which is independent of Atg5 (Collins et al., 2009).

L. monocytogenes can replicate in LAMP1-positive spacious Listeria-containing phagosomes (SLAPs), the formation of which is dependent on both the bacterial virulence toxin listeriolysin $O$ and host autophagy (Birmingham et al., 2008). Interestingly, the listerial virulence factor act $A$ triggers host protein recruitment and actin polymerization, which propels bacteria from one cell to another (Cossart, 2000) and prevents autophagic recognition of bacteria within the cytosol (Yoshikawa et al., 2009). L. monocytogenes expressing-actA mutants that cannot recruit host proteins for actin polymerization 
are eventually ubiquitinated, and then recognized by the autophagy machinery through recruitment of p62 (also known as SQSTM1) and LC3 (Yoshikawa et al., 2009). Notably, L. monocytogenes expressing listeriolysin $\mathrm{O}$, the pore-forming cytolysin, can escape into the cytosol, where $L$. monocytogenes can be targeted for antibacterial autophagy (Huang and Brumell, 2009). Moreover, L. monocytogenes utilizes several virulence mechanisms to escape from host autophagy and grow slowly in specialized niches such as SLAPs, which results in the promotion of bacterial replication and chronic infection (Birmingham et al., 2008; Huang and Brumell, 2009). Recent studies by Dortet et al. have shown that $L$. monocytogenes hijacks the host major vault protein through interaction with $\mathrm{InIK}$, a listerial virulence factor, thus resulting in escape from autophagic recognition and intracellular survival (Dortet et al., 2011).

\section{Bacterial survival and replication inside the pathogen-containing compartments: Mycobacteria, Legionella, Porphyromonas, Coxiella, and Brucella}

$M$. tuberculosis is an enormously successful intracellular pathogen that causes tuberculosis. $M$. tuberculosis is one of the best examples of bacterial survival and replication inside host phagocytes through escape from host immune pathways (Jo, 2010). M. tuberculosis can achieve persistent infection within the hostile environment of the macrophage through rapid transcriptional responses that counteract host immune processes, such as antigen presentation and pro-inflammatory cytokine secretion (Huynh et al., 2011). Arrest of phagosomal maturation into a phagolysosome by M. tuberculosis is a central pathway to overcome host defense and provides these bacteria with a strategy for survival in host cells (Chua et al., 2004; Huynh et al., 2011). Autophagy pathway activation induced by starvation, inhibition of mTOR pathways, vitamin $D$, and interferon- $\gamma$ contributes to the elimination of intracellular $M$. tuberculosis and phagosomal maturation (Gutierrez et al., 2004; Jo, 2010; Deretic, 2011). A genome-wide siRNA screening identified host factors that regulate intracellular survival of M. tuberculosis (Kumar et al., 2010). Importantly, the core host cell factors are found to be predominantly involved in the regulation of the autophagy process (Kumar et al., 2010). Recent studies showed that the M. tuberculosis eis gene negatively modulates host cell autophagy through ROS-dependent mechanisms (Shin et al., 2010a).

Early activation of the autophagy response seems to be crucial for restriction of Legionella pneumophila, an intracellular bacterial pathogen responsible for an acute form of pneumonia, Legionnaire's disease (Dubuisson and Swanson, 2006). Like M. tuberculosis, Legionella can escape from phagolysosome fusion, and perturb and delay the maturation of autophagosomes into autolysosomes (Campoy and Colombo, 2009a; Joshi and Swanson, 2011). Various pathogens such as Porphyromonas gingivalis, Coxiella burnetii and Brucella abortus use an autophagic transit strategy during infection. These bacteria take some advantage of the autophagic pathway, in terms of intracellular survival and replication (Campoy and Colombo, 2009a). Similarly, the facultative intracellular pathogen Francisella tularensis uses a strategy to delay progression along the autophagic pathway, reenter the endocytic pathway after cytoplasmic replication, and even hijack lysosome exocytosis to exit cells (Checroun et al., 2006; Joshi and Swanson, 2011). Future studies will elucidate more detail of the mechanisms by which bacteria interact with host autophagy pathways, which will shed light not only on bacterial pathogenesis at the molecular level but also assist in the development of new therapeutics against refractory chronic infectious diseases.

\section{Cargo receptor and autophagic targeting to bacteria}

Recent reports have suggested that the autophagy pathway involves selective recognition and degradation of the autophagic cargo (Johansen and Lamark, 2011). In selective autophagy, modification of target proteins or intracellular bacteria with ubiquitin is necessary for its autophagic clearance (Kirkin et al., 2009b). Autophagy receptors including p62/SQSTM1 (sequestome 1), NBR1 (neighbour of Brca1 gene) and NDP52 (nuclear dot protein $52 \mathrm{kDa}$ ) can simultaneously bind intracellular ubiquitinated cargos and the autophagy modifiers, such as microtubule-associated protein LC3 and $\gamma$-aminobutyric acid receptor-associated proteins (GABARAP) (Kirkin et al., 2009b; Kraft et al., 2010). Thus, autophagic receptors can mediate docking of ubiquitin-marked protein aggregates to the autophagosomes to induce selective autophagic degradation of ubiquitinated proteins, organelles, and intracellular bacteria (Kirkin et al., 2009a, 2009b; Thurston et al., 2009).

As reviewed above and elsewhere (Sumpter and Levine, 2010), the cargo receptors p62 and NDP52 can target intracellular bacteria, such as $S$. typhimurium, S. flexneri, and L. monocytogenes, by addition of a molecular tag (such as polyubiquitin) to autophagic machinery (Thurston et al., 
2009; Zheng et al., 2009). Recently, it has been shown that optineurin promotes the selective autophagy of ubiquitin-coated cytosolic Salmonella enterica through phosphorylation of optineurin by protein kinase, TANK binding kinase 1 (Wild et al., 2011). Additionally, an ubiquitin-independent pathway mediated by a lipid second messenger, diacylglycerol, generated from SCV membranes, is essential for the initiation of autophagy against $S$. typhimurium (Shahnazari et al., 2010). Ogawa et al. (2011) revealed that the Atg5 binding partner Tecpr1 (tectonin domain-containing protein) is a cargo receptor for targeting bacterial pathogens to the selective autophagy system in Shigella-infected cells. Therefore, several autophagy receptors, which may interconnect with other pathways or mechanisms involved in autophagy, contribute to the xenophagic elimination of cytosolic bacteria in mammalian cells. The mechanisms by which autophagy receptors activate host defenses against bacterial pathogens other than S. typhimurium remain to be determined.

\section{ROS, keeping a balance between antibacterial autophagy and inflammation}

Intracellular ROS, produced by incomplete reduction of oxygen, can oxidize and damage various macromolecules such as proteins, lipids, and DNA, and eventually cause cell death (Azad et al., 2009; Scherz-Shouval and Elazar, 2011). Accumulating evidence points to a key role for ROS as signaling messengers, including the initiation of autophagy and various intracellular processes (Azad et al., 2009; Scherz-Shouval and Elazar, 2011). Several stimuli and stresses, including nutrient starvation, infection, and other pathological conditions, result in ROS generation (Azad et al., 2009). Cellular ROS is involved in innate immune signaling through the TLR-dependent ASK activation (Yuk et al., 2009a) and NADPH oxidase 2 pathways (Yang et al., 2009). Recently, it was revealed that ROS of mitochondrial origin are a critical factor in the antibacterial response, mediated by interaction with TRAF6 and ECSIT (evolutionarily conserved signaling intermediate in Toll pathways), which are themselves involved in the mitochondrial respiratory chain assembly (West et al., 2011).

Interestingly, these are generally accepted as activating signals for autophagy in a variety of physiological and pathological settings (Azad et al., 2009; Scherz-Shouval and Elazar, 2011). Notably, TLR signaling from phagosomes can lead to initiation of antibacterial autophagy through generation of NAPDH oxidase NOX2-dependent ROS (Huang and Brumell, 2009). Excessive ROS of cellular and mitochondrial origin is associated with activation of proinflammatory responses, autophagy, and cell death in macrophages infected with enhanced intracellular survival (eis)-deficient mycobacteria (Shin et al., 2010a).

In contrast, recent evidence suggests that autophagy is also essential for prevention of excessive inflammation. Depletion of the autophagy proteins LC3B and beclin 1 significantly enhances NLRP3dependent inflammasome activation and IL-1 $\beta$ and IL-18 levels, and increases susceptibility to LPSinduced septic shock in vivo (Nakahira et al., 2011). Intriguingly, in this case, mitochondrial ROS are also thought to be important for mediating inflammatory responses, even in the autophagy-deficient host (Nakahira et al., 2011). This effect was dependent on the release of mitochondrial DNA into the cytosol and increased mitochondrial ROS generation (Nakahira et al., 2011). Thus, it seems that coordinated interplay between autophagy activation and ROS-dependent signaling is necessary for the homeostatic regulation of innate defense and inflammation.

\section{Concluding remarks}

Since the roles of autophagy have been revealed in host immune responses, a number of recent in vitro, in vivo, and molecular studies have provided evidence of selective autophagic degradation of bacteria and viruses (xenophagy). Moreover, rapid advances have been made in elucidating the mechanisms underlying the cell's defensive response to intracellular bacteria. In this review we have emphasized that numerous bacterial pathogens possess multiple strategies for avoiding or circumventing host defense pathways to ensure their survival and replication within the host. Thus, it is not surprising that autophagy pathways cross talk with innate immunity to contribute to the combat of invading pathogens. Various immune effectors have been identified as cooperating with and regulating the autophagic responses against invading bacterial infection, although additional host factors and detailed mechanisms remain to be delineated. Here, we propose that the host autophagy system has evolved strategies to manipulate certain autophagic adaptors or cargo receptors to promote autophagy targeting to bacteria, proteins, and damaged organelles. Several important questions related to the extended and distinct roles of immune effectors or cargo receptors, i.e., whether they are involved in defense against specific pathogens or general microbial invaders, should be answered in future studies. Current and future revelations regarding 
the interplay between host autophagy and its evasion by microbial pathogens will provide opportunities for the development of novel antibacterial agents.

\section{Acknowledgements}

We are indebted to both current and past members of our laboratory for the discussions and investigations that contributed to this article. This research was supported by a National Research Foundation of Korea (NRF) grant funded by the Korea government (MEST) through the Infection Signaling Network Research Center (2011-0006228) at Chungnam National University.

\section{References}

Azad AF, Beard CB. Rickettsial pathogens and their arthropod vectors. Emerg Infect Dis 1998;4:179-86

Azad MB, Chen Y, Gibson SB. Regulation of autophagy by reactive oxygen species (ROS): implications for cancer progression and treatment. Antioxid Redox Signal 2009; 11:777-90

Birmingham CL, Smith AC, Bakowski MA, Yoshimori T, Brumell JH. Autophagy controls Salmonella infection in response to damage to the Salmonella-containing vacuole. J Biol Chem 2006;281:11374-83

Birmingham CL, Canadien V, Kaniuk NA, Steinberg BE, Higgins DE, Brumell JH. Listeriolysin $\mathrm{O}$ allows Listeria monocytogenes replication in macrophage vacuoles. Nature 2008;451:350-4

Brodsky IE, Medzhitov R. Targeting of immune signaling networks by bacterial pathogens. Nat Cell Biol 2009; $11: 521-6$

Campoy E, Colombo MI. Autophagy in intracellular bacterial infection. Biochim Biophys Acta 2009a;1793:1465-77

Campoy E, Colombo MI. Autophagy subversion by bacteria. Curr Top Microbiol Immunol 2009b;335:227-50

Cederlund A, Gudmundsson GH, Agerberth B. Antimicrobial peptides important in innate immunity. FEBS J 2011;278: 3942-51

Cemma M, Kim PK, Brumell JH. The ubiquitin-binding adaptor proteins p62/SQSTM1 and NDP52 are recruited independently to bacteria-associated microdomains to target Salmonella to the autophagy pathway. Autophagy 2011;7:341-5

Chang YP, Tsai CC, Huang WC, Wang CY, Chen CL, Lin YS, Kai JI, Hsieh CY, Cheng YL, Choi PC, Chen SH, Chang SP, Liu HS, Lin CF. Autophagy facilitates IFN- $\gamma$-induced Jak2-STAT1 activation and cellular inflammation. J Biol Chem 2010;285:28715-22

Checroun C, Wehrly TD, Fischer ER, Hayes SF, Celli J. Autophagy-mediated reentry of Francisella tularensis into the endocytic compartment after cytoplasmic replication. Proc Natl Acad Sci USA 2006;103:14578-83
Chua J, Vergne I, Master S, Deretic V. A tale of two lipids: Mycobacterium tuberculosis phagosome maturation arrest. Curr Opin Microbiol 2004;7:71-7

Collins CA, De Mazière $A$, van Dijk $S$, Carlsson $F$, Klumperman J, Brown EJ. Atg5-independent sequestration of ubiquitinated mycobacteria. PLoS Pathog 2009;5: e1000430

Cooney R, Baker J, Brain O, Danis B, Pichulik T, Allan P, Ferguson DJ, Campbell BJ, Jewell D, Simmons A. NOD2 stimulation induces autophagy in dendritic cells influencing bacterial handling and antigen presentation. Nat Med 2010; 16:90-7

Cooper AM, Mayer-Barber KD, Sher A. Role of innate cytokines in mycobacterial infection. Mucosal Immunol 2011; 4:252-60

Cossart P. Actin-based motility of pathogens: the Arp2/3 complex is a central player. Cell Microbiol 2000;2:195-205

Delgado MA, Deretic V. Toll-like receptors in control of immunological autophagy. Cell Death Differ 2009;16: 976-83

Deretic V. Multiple regulatory and effector roles of autophagy in immunity. Curr Opin Immunol 2009;21:53-62

Deretic V. Autophagy in infection. Curr Opin Immunol 2010; 22:252-62

Deretic V. Autophagy in immunity and cell-autonomous defense against intracellular microbes. Immunol Rev 2011; 240:92-104

Deretic V, Levine B. Autophagy, immunity, and microbial adaptations. Cell Host Microbe 2009;5:527-49

Djavaheri-Mergny M, Amelotti M, Mathieu J, Besancon F, Bauvy $C$, Souquère $S$, Pierron $G$, Codogno P. NF-kappaB activation represses tumor necrosis factor- $\alpha$-induced autophagy. J Biol Chem 2006;281:30373-82

Dortet L, Mostowy S, Louaka AS, Gouin E, Nahori MA, Wiemer EA, Dussurget O, Cossart P. Recruitment of the major vault protein by InIK: a Listeria monocytogenes strategy to avoid autophagy. PLoS Pathog 2011;7: e1002168

Dubuisson JF, Swanson MS. Mouse infection by Legionella, a model to analyze autophagy. Autophagy 2006;2:179-82

Dupont N, Lacas-Gervais S, Bertout J, Paz I, Freche B, Van Nhieu GT, van der Goot FG, Sansonetti PJ, Lafont F. Shigella phagocytic vacuolar membrane remnants participate in the cellular response to pathogen invasion and are regulated by autophagy. Cell Host Microbe 2009;6:137-49

Furuta N, Yoshimori T, Amano A. Mediatory molecules that fuse autophagosomes and lysosomes. Autophagy 2010; 6:417-8

Gutierrez MG, Master SS, Singh SB, Taylor GA, Colombo MI, Deretic V. Autophagy is a defense mechanism inhibiting BCG and Mycobacterium tuberculosis survival in infected macrophages. Cell 2004;119:753-66

Harris J, De Haro SA, Master SS, Keane J, Roberts EA, Delgado M, Deretic V. T helper 2 cytokines inhibit autophagic control of intracellular Mycobacterium tuberculosis. Immunity 2007;27:505-17 
Harris J, Master SS, De Haro SA, Delgado M, Roberts EA, Hope JC, Keane J, Deretic V. Th1-Th2 polarisation and autophagy in the control of intracellular mycobacteria by macrophages. Vet Immunol Immunopathol 2009;128:37-43

Huang $\mathrm{J}$, Brumell $\mathrm{JH}$. Autophagy in immunity against intracellular bacteria. Curr Top Microbiol Immunol 2009; 335:189-215

Huynh KK, Joshi SA, Brown EJ. A delicate dance: host response to mycobacteria. Curr Opin Immunol 2011; 23:464-72

Ichimura Y, Kumanomidou T, Sou YS, Mizushima T, Ezaki J, Ueno T, Kominami E, Yamane T, Tanaka K, Komatsu M. Structural basis for sorting mechanism of p62 in selective autophagy. J Biol Chem 2008;283:22847-57

Jo EK. Innate immunity to mycobacteria: vitamin D and autophagy. Cell Microbiol 2010;12:1026-35

Jo EK, Yang CS, Choi $\mathrm{CH}$, Harding CV. Intracellular signalling cascades regulating innate immune responses to Mycobacteria: branching out from Toll-like receptors. Cell Microbiol 2007;9:1087-98

Johansen T, Lamark T. Selective autophagy mediated by autophagic adapter proteins. Autophagy 2011;7:279-96

Joshi AD, Swanson MS. Secrets of a successful pathogen: Legionella resistance to progression along the autophagic pathway. Front Microbiol 2011;2:138

Kim BH, Shenoy AR, Kumar P, Das R, Tiwari S, MacMicking JD. A family of IFN- $\gamma$-inducible $65-k D$ GTPases protects against bacterial infection. Science 2011;332:717-21

Kirkin V, Lamark T, Sou YS, Bjørkøy G, Nunn JL, Bruun JA, Shvets E, McEwan DG, Clausen TH, Wild P, Bilusic I, Theurillat JP, Øvervatn A, Ishii T, Elazar Z, Komatsu M, Cikic I, Johansen T. A role for NBR1 in autophagosomal degradation of ubiquitinated substrates. Mol Cell 2009a; 33:505-16

Kirkin V, McEwan DG, Novak I, Dikic I. A role for ubiquitin in selective autophagy. Mol Cell 2009b;34:259-69

Knodler LA, Celli J, Finlay BB. Pathogenic trickery: deception of host cell processes. Nat Rev Mol Cell Biol 2001;2:578-88

Kraft C, Peter M, Hofmann K. Selective autophagy: ubiquitin-mediated recognition and beyond. Nat Cell Biol 2010;12:836-41

Kumar D, Nath L, Kamal MA, Varshney A, Jain A, Singh S, Rao KV. Genome-wide analysis of the host intracellular network that regulates survival of Mycobacterium tuberculosis. Cell 2010;140:731-43

Lee HM, Shin DM, Yuk JM, Shi G, Choi DK, Lee SH, Huang SM, Kim JM, Kim CD, Lee JH, Jo EK. Autophagy negatively regulates keratinocyte inflammatory responses via scaffolding protein p62/SQSTM1. J Immunol 2011;186: 1248-58

Levine B, Deretic V. Unveiling the roles of autophagy in innate and adaptive immunity. Nat Rev Immunol 2007;7:767-77

Levine B, Mizushima N, Virgin HW. Autophagy in immunity and inflammation. Nature 2011;469:323-35
Ling YM, Shaw MH, Ayala C, Coppens I, Taylor GA, Ferguson DJ, Yap GS. Vacuolar and plasma membrane stripping and autophagic elimination of Toxoplasma gondii in primed effector macrophages. J Exp Med 2006;203:2063-71

Liu PT, Stenger S, Li H, Wenzel L, Tan BH, Krutzik SR, Ochoa MT, Schauber J, Wu K, Meinken C, Kamen DL, Wagner M, Bals R, Steinmeyer A, Zügel U, Gallo RL, Eisenberg D, Hewison M, Hollis BW, Adams JS, Bloom BR, Modlin RL. Toll-like receptor triggering of a vitamin D-mediated human antimicrobial response. Science 2006;311:1770-3

Manavalan B, Basith S, Choi S. Similar structures but different roles - an updated perspective on TLR structures. Front Physiol 2011;2:41

Mizushima N, Levine B, Cuervo AM, Klionsky DJ. Autophagy fights disease through cellular self-digestion. Nature 2008; 451:1069-75

Nakagawa I, Amano A, Mizushima N, Yamamoto A, Yamaguchi H, Kamimoto T, Nara A, Funao J, Nakata M, Tsuda K, Hamada S, Yoshimori T. Autophagy defends cells against invading group A Streptococcus. Science 2004; 306:1037-40

Nakahira K, Haspel JA, Rathinam VA, Lee SJ, Dolinay T, Lam HC, Englert JA, Rabinovitch M, Cernadas M, Kim HP, Fitzgerald KA, Ryter SW, Choi AM. Autophagy proteins regulate innate immune responses by inhibiting the release of mitochondrial DNA mediated by the NALP3 inflammasome. Nat Immunol 2011;12:222-30

Ogawa M, Yoshimori T, Suzuki T, Sagara H, Mizushima N, Sasakawa C. Escape of intracellular Shigella from autophagy. Science 2005;307:727-31

Ogawa M, Yoshikawa Y, Kobayashi T, Mimuro H, Fukumatsu M, Kiga K, Piao Z, Ashida H, Yoshida M, Kakuta S, Koyama T, Goto Y, Nagatake T, Nagai S, Kiyono H, Kawalec M, Reichhart JM, Sasakawa C. A Tecpr1-dependent selective autophagy pathway targets bacterial pathogens. Cell Host Microbe 2011;9:376-89

Orvedahl A, Levine B. Eating the enemy within: autophagy in infectious diseases. Cell Death Differ 2009;16:57-69

Pankiv S, Clausen TH, Lamark T, Brech A, Bruun JA, Outzen $H, \varnothing v e r v a t n A, B j ø r k ø y ~ G$, Johansen T. p62/SQSTM1 binds directly to Atg8/LC3 to facilitate degradation of ubiquitinated protein aggregates by autophagy. J Biol Chem 2007;282: 24131-45

Pattingre S, Bauvy C, Carpentier S, Levade T, Levine B, Codogno P. Role of JNK1-dependent Bcl-2 phosphorylation in ceramide-induced macroautophagy. J Biol Chem 2009; 284:2719-28

Rikihisa Y. Glycogen autophagosomes in polymorphonuclear leukocytes induced by rickettsiae. Anat Rec 1984;208: 319-27

Sakurai A, Maruyama F, Funao J, Nozawa T, Aikawa C, Okahashi N, Shintani S, Hamada S, Ooshima T, Nakagawa I. Specific behavior of intracellular Streptococcus pyogenes that has undergone autophagic degradation is associated with bacterial streptolysin $\mathrm{O}$ and host small $\mathrm{G}$ proteins Rab5 and Rab7. J Biol Chem 2010;285:22666-75 
Scherz-Shouval R, Elazar Z. Regulation of autophagy by ROS: physiology and pathology. Trends Biochem Sci 2011; 36:30-8

Shahnazari S, Brumell JH. Mechanisms and consequences of bacterial targeting by the autophagy pathway. Curr Opin Microbiol 2011;14:68-75

Shahnazari S, Yen WL, Birmingham CL, Shiu J, Namolovan A, Zheng YT, Nakayama K, Klionsky DJ, Brumell JH. A diacylglycerol-dependent signaling pathway contributes to regulation of antibacterial autophagy. Cell Host Microbe 2010;8:137-46

Shi CS, Kehrl JH. MyD88 and Trif target Beclin 1 to trigger autophagy in macrophages. J Biol Chem 2008;283: 33175-82

Shi CS, Kehrl JH. TRAF6 and A20 regulate lysine 63-linked ubiquitination of Beclin-1 to control TLR4-induced autophagy. Sci Signal 2010;3:ra42

Shin DM, Jeon BY, Lee HM, Jin HS, Yuk JM, Song CH, Lee $\mathrm{SH}$, Lee ZW, Cho SN, Kim JM, Friedman RL, Jo EK. Mycobacterium tuberculosis eis regulates autophagy, inflammation, and cell death through redox-dependent signaling. PLoS Pathog 2010a;6:e1001230

Shin DM, Yuk JM, Lee HM, Lee SH, Son JW, Harding CV, Kim JM, Modlin RL, Jo EK. Mycobacterial lipoprotein activates autophagy via TLR2/1/CD14 and a functional vitamin D receptor signaling. Cell Microbiol 2010b;12:1648-65

Silverman DJ, Wisseman CL, Jr. In vitro studies of rickettsiahost cell interactions: ultrastructural changes induced by Rickettsia rickettsii infection of chicken embryo fibroblasts. Infect Immun 1979;26:714-27

Singh SB, Davis AS, Taylor GA, Deretic V. Human IRGM induces autophagy to eliminate intracellular mycobacteria. Science 2006;313:1438-41

Sonawane A, Santos JC, Mishra BB, Jena P, Progida C, Sorensen OE, Gallo R, Appelberg R, Griffiths G. Cathelicidin is involved in the intracellular killing of mycobacteria in macrophages. Cell Microbiol 2011;13:1601-17

Stamm LM, Brown EJ. Mycobacterium marinum: the generalization and specialization of a pathogenic mycobacterium. Microbes Infect 2004;6:1418-28

Sumpter R Jr, Levine B. Autophagy and innate immunity: triggering, targeting and tuning. Semin Cell Dev Biol 2010; 21:699-711

Takeda K, Akira S. TLR signaling pathways. Semin Immunol 2004;16:3-9

Takeuchi O, Akira S. Pattern recognition receptors and inflammation. Cell 2010;140:805-20

Thurston TL, Ryzhakov G, Bloor S, von MN, Randow F. The TBK1 adaptor and autophagy receptor NDP52 restricts the proliferation of ubiquitin-coated bacteria. Nat Immunol 2009; 10:1215-21

von Muhlinen N, Thurston T, Ryzhakov G, Bloor S, Randow F. NDP52, a novel autophagy receptor for ubiquitindecorated cytosolic bacteria. Autophagy 2010;6:288-9

Walker DH. Rickettsiae and rickettsial infections: the current state of knowledge. Clin Infect Dis 2007;45 (Suppl 1):S39-44

Walker DH, Popov VL, Crocquet-Valdes PA, Welsh CJ, Feng HM. Cytokine-induced, nitric oxide-dependent, intracellular antirickettsial activity of mouse endothelial cells. Lab Invest 1997;76:129-38

Wei Y, Pattingre S, Sinha S, Bassik M, Levine B. JNK1mediated phosphorylation of $\mathrm{Bcl}-2$ regulates starvationinduced autophagy. Mol Cell 2008;30:678-88

West AP, Brodsky IE, Rahner C, Woo DK, ErdjumentBromage H, Tempst P, Walsh MC, Choi Y, Shadel GS, Ghosh $S$. TLR signalling augments macrophage bactericidal activity through mitochondrial ROS. Nature 2011;472:476-80

Wild P, Farhan H, McEwan DG, Wagner S, Rogov VV, Brady NR, Richter B, Korac J, Waidmann O, Choudhary C, Dötsch V, Bumann D, Dikic I. Phosphorylation of the autophagy receptor optineurin restricts Salmonella growth. Science 2011;333:228-33

Xu Y, Jagannath C, Liu XD, Sharafkhaneh A, Kolodziejska $\mathrm{KE}$, Eissa NT. Toll-like receptor 4 is a sensor for autophagy associated with innate immunity. Immunity 2007;27:135-44

Yang CS, Shin DM, Kim KH, Lee ZW, Lee CH, Park SG, Bae YS, Jo EK. NADPH oxidase 2 interaction with TLR2 is required for efficient innate immune responses to mycobacteria via cathelicidin expression. J Immunol 2009; 182:3696-705

Yoshikawa Y, Ogawa M, Hain T, Yoshida M, Fukumatsu M, Kim M, Mimuro H, Nakagawa I, Yanagawa T, Ishii T, Kakizuka A, Sztul E, Chakraborty T, Sasakawa C. Listeria monocytogenes ActA-mediated escape from autophagic recognition. Nat Cell Biol 2009;11:1233-40

Yuk JM, Shin DM, Lee HM, Yang CS, Jin HS, Kim KK, Lee ZW, Lee SH, Kim JM, Jo EK. Role of apoptosis-regulating signal kinase 1 in innate immune responses by Mycobacterium bovis bacillus Calmette-Guerin. Immunol Cell Biol 2009a;87:100-7

Yuk JM, Shin DM, Lee HM, Yang CS, Jin HS, Kim KK, Lee ZW, Lee SH, Kim JM, Jo EK. Vitamin D3 induces autophagy in human monocytes/macrophages via cathelicidin. Cell Host Microbe 2009b;6:231-43

Zheng YT, Shahnazari S, Brech A, Lamark T, Johansen T, Brumell JH. The adaptor protein p62/SQSTM1 targets invading bacteria to the autophagy pathway. J Immunol 2009;183:5909-16 